\title{
Clinical Correlation between Real-Time Endocytoscopy, Confocal Endomicroscopy, and Histopathology in the Central Airways
}

\author{
Pallav L. Shah ${ }^{\mathrm{a}-\mathrm{c}}$ Samuel V. Kemp ${ }^{a}$ Richard C. Newton ${ }^{d}$ Daniel S. Elson ${ }^{d}$ \\ Andrew G. Nicholson ${ }^{a}$ Guang-Zhong Yang ${ }^{d}$ \\ ${ }^{a}$ The National Institute for Health Research Respiratory Biomedical Research Unit, Royal Brompton and \\ Harefield NHS Foundation Trust, and Imperial College, ${ }^{b}$ Chelsea and Westminster Hospital, ${ }^{c}$ Imperial College, and \\ dThe Hamlyn Centre for Robotic Surgery, Imperial College, London, UK
}

\section{Key Words}

Bronchoscopy · Fluorescence bronchoscopy .

Endocytoscopy - Probe-based confocal endomicroscopy .

Dysplasia $\cdot$ Bronchial carcinoma

\begin{abstract}
Background: Lung cancer is one of the commonest malignancies with a worldwide incidence of 1.6 million cases each year. Although the main aetiological factor has been identified (cigarette smoking), the progression of lung cancer from early changes such as dysplasia through to cancer is still not fully understood. Furthermore, current research techniques are reliant on obtaining tissue biopsies, a process that alters the natural history of the very process under investigation. Hence, there is a need for developing optical biopsy techniques. Objectives: To prospectively evaluate the feasibility of endocytoscopy and confocal endomicroscopy in the detection of malignant and pre-malignant changes in the airways. Methods: Findings with endocytoscopy and endomicroscopy were compared with conventional biopsies obtained from the same areas in 25 patients undergoing bronchoscopy for evaluation of endobronchial abnormalities and in 5 healthy control subjects. Results: Endocytoscopy was technically more difficult, and interpretable imag-
\end{abstract}

es were only obtained in 21 of the patients evaluated, and hence, complete information including histopathological information was available in 21 patients. Endocytoscopy appeared to correlate with the histopathological findings on tissue biopsy, and was able to distinguish normal epithelium from dysplasia and carcinoma. Confocal endomicroscopy was a more reliable technique with adequate visual information obtained in all patients examined but was unable to distinguish between dysplasia and carcinoma. Conclusion: This feasibility study suggests that endocytoscopy may have the potential to fulfil the role of optical biopsy in the evaluation of the pathogenesis of lung cancer.

(c) 2016 S. Karger AG, Basel

\section{Introduction}

Lung cancer is one of the commonest malignancies with a worldwide incidence estimated at 1.6 million, with about $1,095,200$ cases in males and 513,600 in females [1]. Lung cancer accounts for $20 \%$ of all cancers in men with a lifetime risk of 1 in 13 , and $12 \%$ of all cancers in women with a lifetime risk of 1 in 23 [2]. Squamous cell carcinoma (SQCC) is the second most common type of lung cancer after adenocarcinoma. Smoking is the main aetiological

\section{KARGER}

(c) 2016 S. Karger AG, Basel

E-Mail karger@karger.com

www.karger.com/res
Dr. Samuel V. Kemp

Royal Brompton Hospital

Sydney Street

London SW3 6NP (UK)

E-Mail s.kemp@ rbht.nhs.uk 


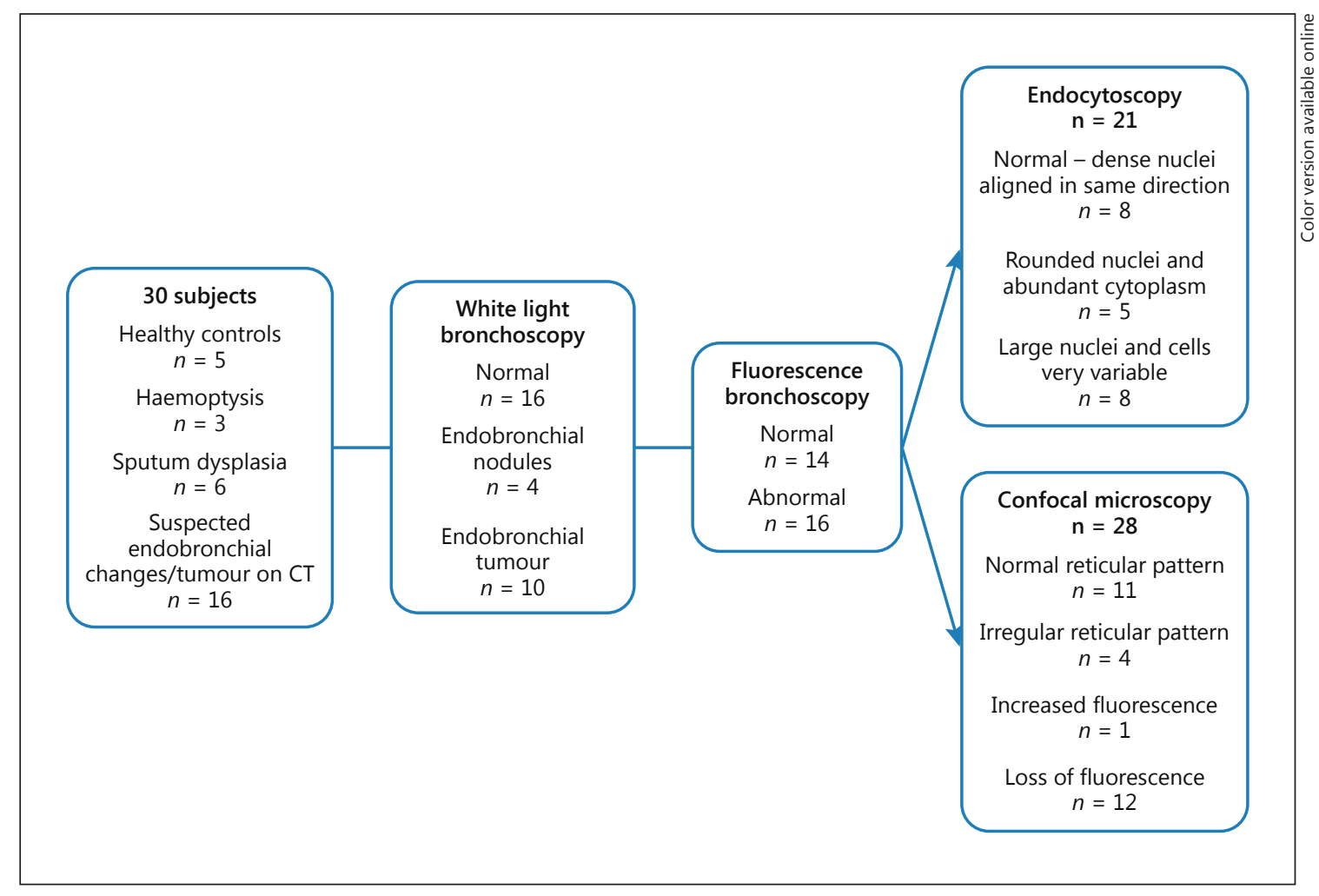

Fig. 1. Flow chart illustrating characteristics of subjects recruited and findings obtained by white light bronchoscopy, fluorescence bronchoscopy, confocal endomicroscopy, and endocytoscopy.

factor for SQCC, as it exposes the lung to numerous carcinogenic substances which induce direct and indirect damage to DNA, RNA, and proteins. A general assumption is that SQCC typically develops in a stepwise manner from squamous metaplasia through dysplasia and carcinoma in situ (CIS), and finally to invasive disease [3-5]. However, some longitudinal studies have suggested that this natural history may be more complex and there may be changes that are out of step, with both progression and regression of pathological changes seen within lesions over time [6].

One of the major limitations of some of these studies is that the natural history is altered by the process of obtaining a tissue biopsy. Indeed, one study reported complete removal of the dysplastic areas following forceps biopsy [7]. Hence, there is a need to develop optical biopsy tools, where histopathological information can be obtained from the surface epithelium without the need for a tissue biopsy [8]. We have prospectively evaluated 2 novel optical imaging techniques, endocytoscopy and confocal endomicroscopy, and how findings obtained with these 2 techniques correlate with conventional biopsies obtained from the same areas.

\section{Methods}

Patients undergoing bronchoscopy for diagnostic purposes following an episode of haemoptysis or those with a suspected endobronchial neoplasm were enrolled (Fig. 1). The purpose of the study was to evaluate the utility of 2 optical imaging techniques, confocal microscopy and endocytoscopy, in a spectrum of patients with normal endobronchial epithelium through to those with areas of severe dysplasia and invasive carcinoma.

The procedure was conducted under local anaesthesia with or without midazolam. Flexible bronchoscopy was first performed using a video-bronchoscope (BF-F260; Olympus Medical, Tokyo, Japan) with both white light and fluorescence functionality. The trachea, main bronchi, and segmental bronchi were first examined with white light bronchoscopy, and the procedure was then repeated under blue light (fluorescence mode). The location and extent of any abnormalities were systematically noted using standard nomenclature.

\section{Confocal Endomicroscopy}

Confocal endomicroscopy was performed using a probe-based confocal laser endomicroscope (pCLE, Cellvizio ${ }^{\circledR}$-Lung; Mauna Kea Technologies, Paris, France). The imaging unit delivers laser light at a wavelength of $488 \mathrm{~nm}$, whilst the confocal probe (which measures $1.4 \mathrm{~mm}$ in diameter) is inserted through the instrument channel of the bronchoscope. This is a contact probe, and 
therefore, it was gently apposed to the airways and the image displayed on a monitor. The image produced had a $600-\mu \mathrm{m}$ diameter field of view with 9 frames per second, imaging to a depth of up to $50 \mu \mathrm{m}$. The system captures still images, movie clips, and still mosaics where adjacent images are stapled together to increase the field of view. Normal airways in a patient were first examined followed by areas identified as abnormal by white light and fluorescence imaging.

\section{Endocytoscopy}

After completing the examination with the pCLE probe, the bronchoscope was removed and the patient was intubated with a prototype hybrid endocytoscope (XEC-300-U/F; Olympus, Tokyo, Japan). This integrated bronchoscope provides both normal and endocytoscope functions. About $0.5 \mathrm{ml}$ of $2 \%$ methylene blue was instilled around the area of interest and the bronchoscope was gently apposed. The image produced was magnified 570 times, with a resolution of $4.2 \mu \mathrm{m}$, a field of view of $300 \times 300 \mu \mathrm{m}$, and a depth of observation from 0 to $30 \mu \mathrm{m}$. Control images were first obtained by examining an area of normal epithelium. Again, both still and video image findings were recorded. The process was repeated by applying the endocytoscope to the abnormal area identified at fluorescence and white light bronchoscopy.

\section{Histology}

Bronchial biopsies were first obtained from a normal control area, usually the right middle lobe carina (RC2), and then at least 2 bronchial biopsies were obtained from each abnormal area. Histological analysis was performed by 2 expert pathologists after routine haematoxylin and eosin (HE) staining, and in selected cases, routine immunohistochemical staining for pankeratin (MNF116), of 5 - $\mu \mathrm{m}$ sections of formalin-fixed, paraffin-embedded tissue.

The study was approved by the Brompton, Harefield, and NHLI Research Ethics Committee (09/H0708/18), and all patients provided written consent. The trial was registered at www. clinicaltrials.gov (NCT00934778).

\section{Results}

Thirty subjects were recruited for assessment between June 9, 2009 and September 15, 2011. Optical imaging with endocytoscopy, confocal endomicroscopy, and matching histopathological biopsies was available in 21 subjects (Fig. 1). An obvious endobronchial abnormality was visible on white light bronchoscopy in 14 patients. A further 2 patients had abnormal changes only visible during examination in the fluorescence mode.

Adequate endocytological images were obtained in only 21 patients during attempted endocytoscopy. This is a technically challenging procedure as it relies on the endocytoscope being held in a steady position a very short focal distance away from the mucosa $(20-30 \mu \mathrm{m})$. Hence, coughing, breathing movement, and any general movement by the patient or operator affects the quality of the images obtained. In our experience, the best strategy was to try to obtain an adequate video loop and then create stills from these videos which could then be evaluated in more detail. In contrast, apposition of the pCLE probe and acquisition of interpretable pCLE images was a simple and reliable technique, with reproducible images obtained in all patients despite also being a contact imaging technique.

HE staining of normal bronchial mucosal biopsies and examination with a microscope at 200 times magnification revealed the surface lined with intact respiratory, ciliated epithelium lacking atypia or suprabasal mitoses (Fig. 2). In cases of dysplasia, the typical histological features comprised squamous metaplasia with varying degrees of disorganisation of the epithelium, with nuclear atypia and suprabasal mitoses, but with a degree of differentiation towards the epithelial surface. In patients with CIS, the changes were histologically similar to dysplasia, but lacked differentiation in superficial cell layers. The appearances of the surface epithelium in patients with invasive carcinoma were similar to CIS, but demonstrated frank invasion with strands and clusters of atypical cells in the underlying stroma, accompanied by a desmoplastic tissue response.

The appearance of normal endobronchial mucosa with pCLE demonstrates the reticular structure of the elastin skeleton of the endobronchial tree (Fig. 2). These appearances are interspersed by circular defects representing the mucosal pits or glands. In areas of dysplasia, the fluorescence is muted and the reticular structure is lost, with a similar appearance found with both CIS and neoplasia involving the endobronchial mucosa.

Despite the difficulties encountered with endocytoscopy, when adequate images were obtained, the findings appeared to correlate with the histological findings (Fig. 2). Normal mucosa has the appearance of elongated cells with dense nuclei of a uniform appearance which are all aligned in one direction. In patients with dysplasia, the cells appear more rounded with larger rounded nuclei, abundant cytoplasm, and some variability in the ratio of nuclear to cytoplasm volume. The endocytoscopy appearance of carcinoma is more variable than the appearances seen with dysplasia. The cells are more irregular, have a larger amount of nuclear material and a higher nucleus-to-cytoplasm ratio, and necrotic cells may also be present. The appearance of CIS is similar but with fewer necrotic cells and areas of gradation which appear similar to dysplasia.

Two subjects were found to have endobronchial metastases from extrapulmonary tumours. The first was an endobronchial metastasis from a renal cell carcinoma, 


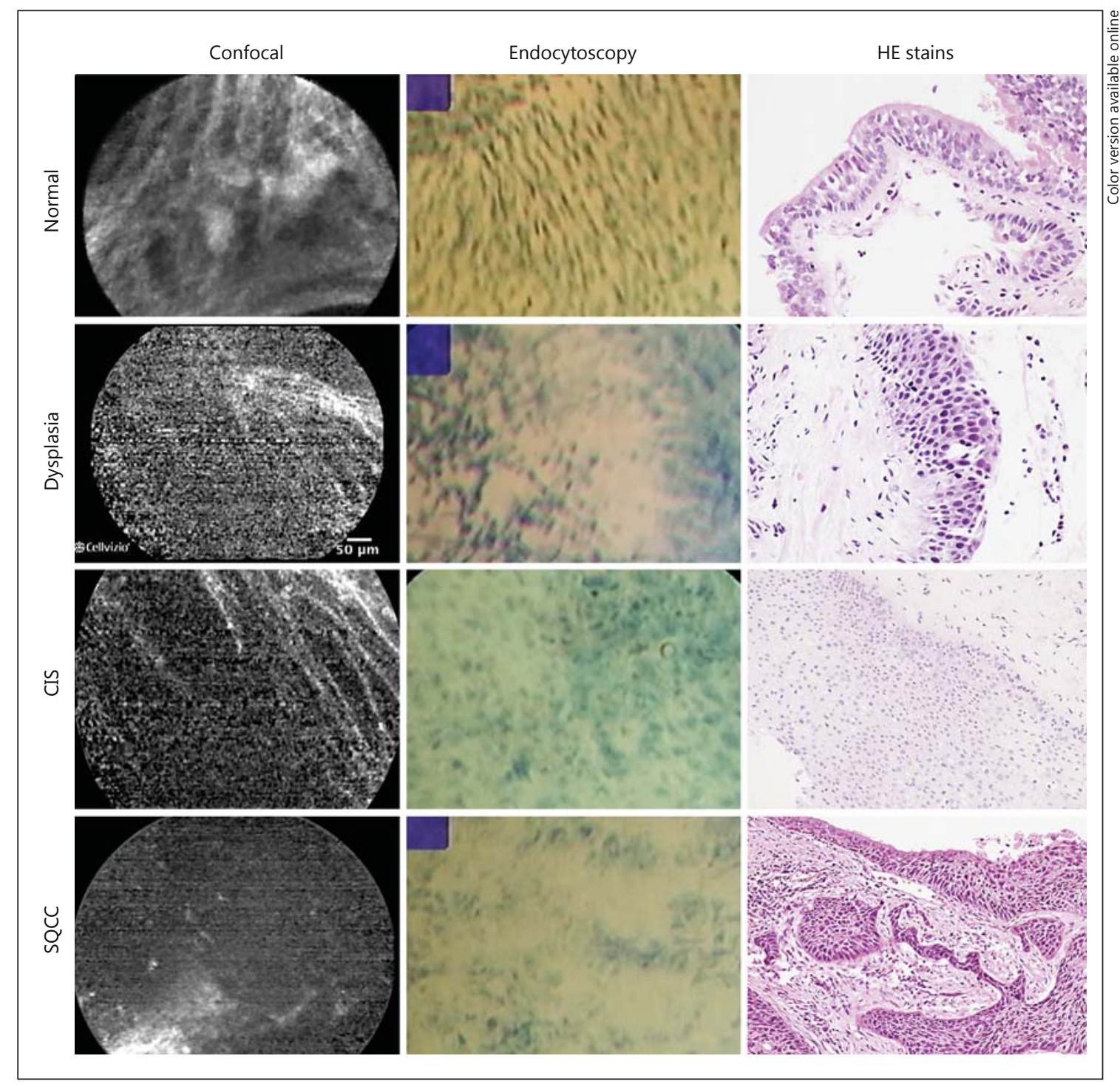

Fig. 2. Confocal endomicroscopy, endocytoscopy, and histological appearances of endobronchial epithelium for the various stages of carcinogenesis: normal epithelium, dysplastic epithelium, CIS, and invasive SQCC. The HEstained sections for normal bronchial mucosa and dysplasia are at 200× magnification, and those for CIS and invasive SQCC are at $100 \times$ magnification.

with scattered nuclei interspersed with amorphous material and ghost-like cellular areas at endocytoscopy (Fig. 3). This correlates closely with the histological appearance of nested tumour cells with ample, clear cytoplasm. The second case was a patient with a metastasis from an osteosarcoma, which on histology demonstrated atypical osteoblastic tumour cells within areas of necrosis, and corresponded to a similarly haphazard pattern on endocytoscopy.

\section{Discussion}

Despite its technical challenges, endocytoscopy appears to show some promise as a technique for optical biopsy. There were distinct changes visible between normal bronchial epithelium, dysplasia, and carcinoma. The findings correlated closely with the histopathological samples. To date, no other optical technique, including fluorescence bronchoscopy and narrow band imaging, has been able to distinguish between dysplasia and 


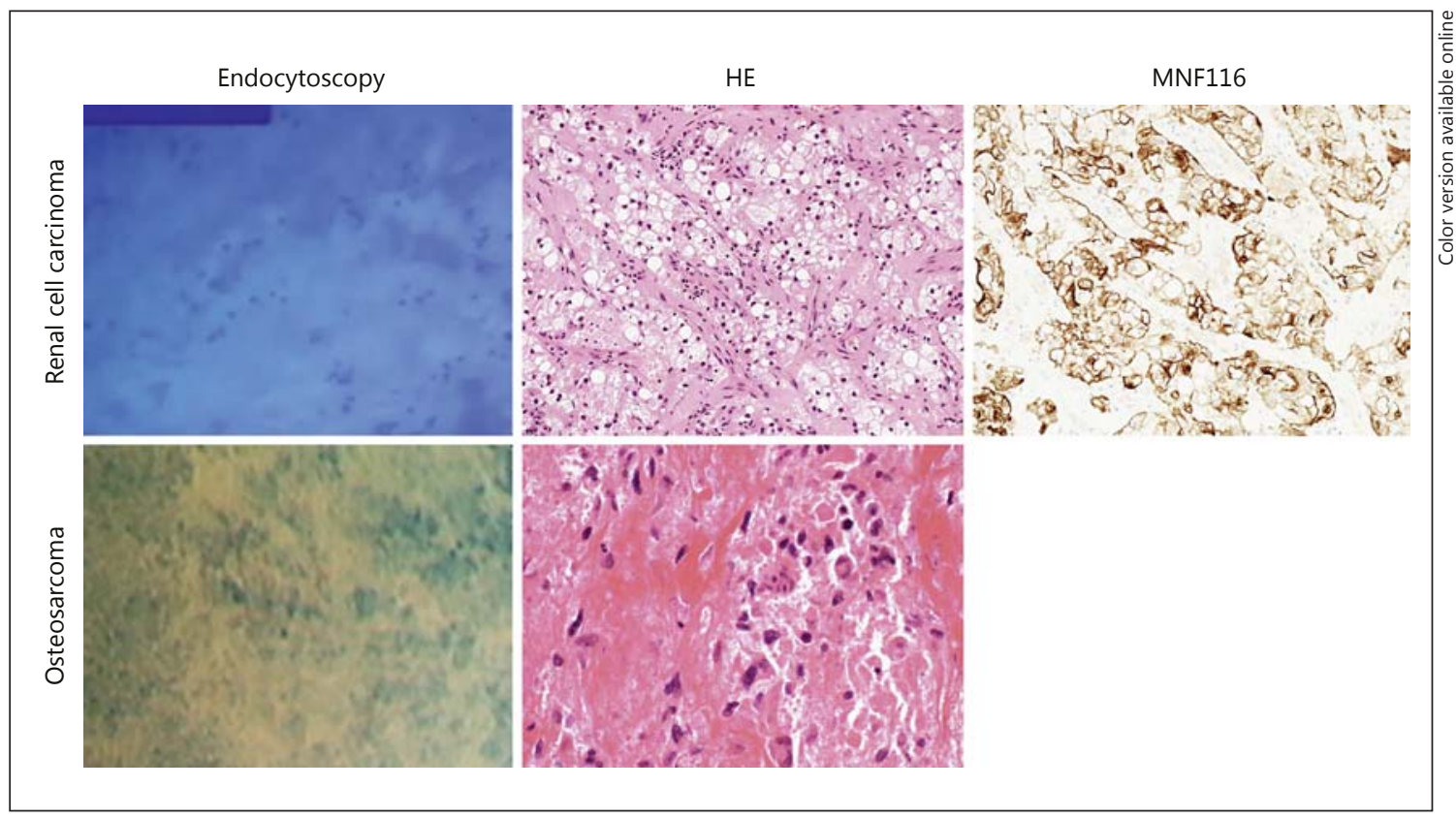

Fig. 3. Endocytoscopy appearance of metastases (renal cell carcinoma and osteosarcoma).

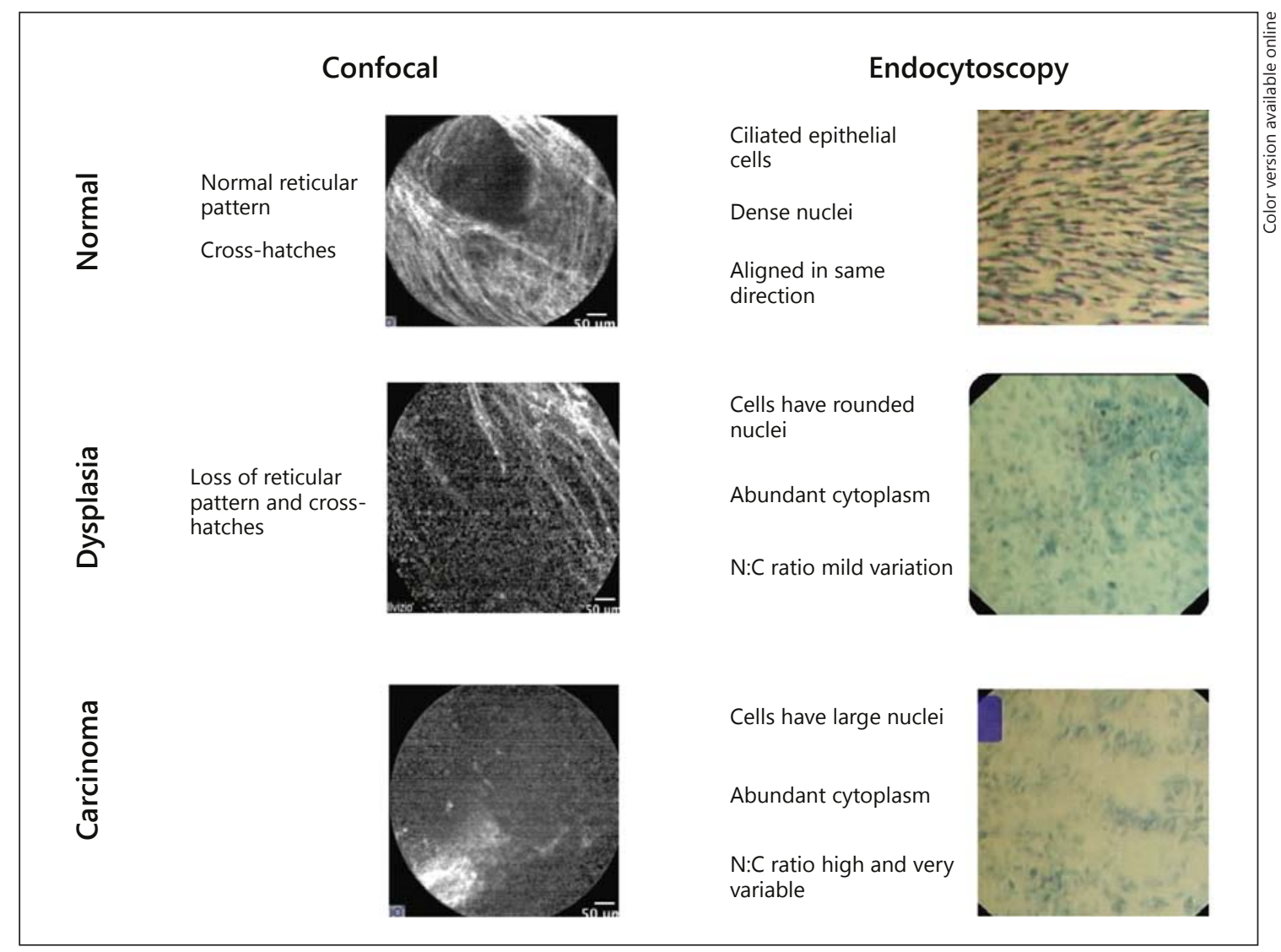

Fig. 4. Comparison of findings between confocal endomicroscopy and endocytoscopy for normal epithelium, dysplasia, and carcinoma. N:C, nucleus-to-cytoplasm ratio.

Real-Time Endocytoscopy, Confocal Endomicroscopy, and Histopathology
Respiration 2017;93:51-57 DOI: $10.1159 / 000452959$ 
carcinoma. Confocal endomicroscopy was able to distinguish between normal bronchial tissue and abnormal bronchial tissue but was unable to distinguish between dysplasia and carcinoma, although 1 report has shown improved image interpretation with the use of topical acriflavine for cell staining at pCLE, with an accuracy of 91\% for detecting malignancy [9]. The comparative findings for the 2 techniques are summarised in Figure 4 . The ability to recognise early carcinoma or CIS is important, as 5 -year survival rates in this group are between 74 and $91 \%$ compared to survival rates for lung cancer as a whole of approximately $15 \%[2,10-12]$.

There is a wealth of literature describing the imaging features and diagnostic accuracy of pCLE and endocytoscopy within the gastrointestinal tract. However, no studies have utilised all available optical techniques at the same setting in vivo in patients. Furthermore, the application of endocytoscopy to evaluate the bronchial epithelium has been very sparse, with the literature limited to 1 case series and 2 case reports using an early generation probe-based endocytoscope [13-15].

The key limitation of this work is the small number of patients evaluated. Endocytoscopy appears to be the more promising technique for distinguishing between dysplasia and carcinoma but is challenging to perform. Very careful and precise manipulation of the bronchoscope is required to hold the tip of the scope about $20-30 \mu \mathrm{m}$ above the airway epithelium in order to obtain adequate quality cytological images of the epithelium. The technique can be difficult to implement during bronchoscopy under conscious sedation, and may be facilitated by general anaesthesia. Furthermore, it is impossible to evaluate the whole of the accessible endobronchial tree, and it can only be used to evaluate areas of interest. The most effective way of utilising endocytoscopy is first to identify abnormal areas with a combination of white light and fluorescence bronchoscopy. Anatomical landmarks should be used to determine the location of the abnormality. After instillation of methylene blue, the endocytoscope is apposed to the epithelial surface close to the area of abnormality and slowly panned across the surface whilst recording the video images, which can be carefully studied after the procedure.

Endocytoscopy remains a research tool, and as such, validation currently requires the taking of endobronchial biopsies. As the technique matures and an evidence base is accumulated, this may obviate the need for biopsies in certain situations. Further developments such as image stabilisation and best picture capture similar to those found in commercial digital cameras may enhance the utility of endocytoscopy. Alternatively, a probe-based system as used for confocal microscopy may be easier to manipulate and hold steady over the bronchial mucosa to obtain good quality images instead of the current integrated bronchoscope/endocytoscope prototype. In combination with accurate navigation techniques, a probebased system may also allow the characterisation of peripheral nodules in situ, something that may become increasingly important with the advent of lung cancer screening.

A further problem with endocytoscopy was the difficulty in evenly staining the bronchial mucosa with methylene blue. The methylene blue liquid tends to pool in patches with uneven staining. Methylene blue is a vital stain that has been shown to be absorbed by abnormal intestinal tissue but not by squamous epithelium [16]. A technique known as chromoendoscopy has been developed, whereby a fine mist is created to evenly stain large areas of mucosa, followed by rinsing and suctioning away any dye that has not been absorbed within a couple of minutes. Any areas that have not been stained may be secondary to squamous metaplasia, and the technique allows more targeted biopsy in Barrett's oesophagus [17]. There is 1 report of selective staining of bronchial tumours with methylene blue [18]. As normal bronchial epithelium does not absorb methylene blue, any selective staining of the bronchial mucosa could be used for targeting endocytoscopy and optimising this technique. There is now a need for bigger blinded studies directly comparing techniques in the same patient population, with the calculation of sensitivities and specificities for the different techniques, and inter-observer agreement values.

\section{Conclusions}

This preliminary study suggests that endocytoscopy is feasible with some utility as an optical biopsy technique, as it is able to distinguish between dysplasia and CIS/carcinoma. It also has the potential to increase our understanding of the natural history of dysplastic airway mucosa and the subsequent development of lung cancer. However, the technique can be difficult to implement during bronchoscopy.

\section{Disclosure Statement}

The authors have no conflicts of interest to disclose.
Shah/Kemp/Newton/Elson/Nicholson/ Yang 


\section{References}

$>1$ Ferlay J, Shin HR, Bray F, et al: Estimates of worldwide burden of cancer in 2008: GLOBOCAN 2008. Int J Cancer 2010;127:28932917.

2 Ferlay J, Shin HR, Bray F, Forman D, Mathers C, Parkin DM: GLOBOCAN 2008 v1.2: Cancer incidence and mortality worldwide: IARC CancerBase No. 10. Lyon, International Agency for Research on Cancer, 2010. http:// globocan.iarc.fr.

$\checkmark 3$ Auerbach O, Stout AP, Hammond EC, et al: Changes in bronchial epithelium in relation to cigarette smoking and in relation to lung cancer. N Engl J Med 1961;265:255-267.

4 Auerbach O, Hammond EC, Garfinkel L: Changes in bronchial epithelium in relation to cigarette smoking, $1955-1960$ vs. 1970 1977. N Engl J Med 1979;300:381-385.

$\checkmark 5$ Saccomanno G, Archer VE, Auerbach O, Saunders RP, Brennan LM: Development of carcinoma of the lung as reflected in exfoliated cells. Cancer 1974;33:256-270.

-6 Breuer RH, Pasic A, Smit EF, et al: The natural course of preneoplastic lesions in bronchial epithelium. Clin Cancer Res 2005;11:537543.
7 Gazdar AF, Park IW, Sood IS: Clonal patches of molecular changes in smoking damaged respiratory epithelium. Lung Cancer 2000; 29:S7.

8 Newton RC, Kemp SV, Shah PL, Elson D, Darzi A, Shibuya K, Mulgrew S, Yang GZ: Progress toward optical biopsy: bringing the microscope to the patient. Lung 2011;189: 111-119.

-9 Fuchs FS, Zirlik S, Hildner K, Schubert J, Vieth M, Neurath MF: Confocal laser endomicroscopy for diagnosing lung cancer in vivo. Eur Respir J 2013;41:1401-1408.

$\checkmark 10$ Cortese DA, Pairolero PC, Bergstralh EJ, et al: Roentgenographically occult lung cancer: a 10-year experience. J Thorac Cardiovasc Surg 1983;86:373-380.

11 Bechtel JJ, Petty TL, Saccomanno G: Five year survival and later outcome of patients with $\mathrm{X}$ ray occult lung cancer detected by sputum cytology. Lung Cancer 2000;30:1-7.

$\checkmark 12$ Lam S, MacAulay C, LeRiche JC, Palcic B: Detection and localization of early lung cancer by fluorescence bronchoscopy. Cancer 2000; 89:2468-2473.
3 Shibuya K, Fujiwara T, Yasufuku K, et al: In vivo microscopic imaging of the bronchial mucosa using an endo-cytoscopy system. Lung Cancer 2011;72:184-190.

14 Kitamura Y, Yamada G, Narita Y, et al: Bronchoscopic observation of endobronchial tumor cells. J Bronchology Interv Pulmonol 2012;19:311-312.

-15 Neumann H, Vieth M, Neurath MF, Fuchs FS: In vivo diagnosis of small-cell lung cancer by endocytoscopy. J Clin Oncol 2011; 29:e131-e132.

16 Fennerty MB, Sampliner RE, McGee DL, Hixon LJ, Garewal HS: Intestinal metaplasia of the stomach identification by a selective mucosal staining technique. Gastrointest Endosc 1992;38:696-698.

17 Canto M, Setrakian S, Willis J, et al: Methylene blue-directed biopsy for improved detection of intestinal metaplasia and dysplasia in Barrett's esophagus: a controlled sequential trial. Gastrointest Endosc 2000;51:560-568.

18 Varoli F, Mariani C, Fascianella A, Cosentino F, Roviaro GC: Vital staining in fiberoptic bronchoscopy. Endoscopy 1986;18:142-143. 